University of Nebraska - Lincoln

DigitalCommons@University of Nebraska - Lincoln

2006

\title{
A measurement of electron-wall interactions using transmission diffraction from nanofabricated gratings
}

\author{
Brett E. Barwick \\ University of Nebraska-Lincoln, brett.barwick@trincoll.edu \\ Glen Gronniger \\ University of Nebraska - Lincoln \\ Lu Yuan \\ University of Nebraska - Lincoln \\ Sy-Hwang Liou \\ University of Nebraska-Lincoln, sliou@unl.edu \\ Herman Batelaan \\ University of Nebraska - Lincoln, hbatelaan@unl.edu
}

Follow this and additional works at: https://digitalcommons.unl.edu/mrsecfacpubs

Part of the Materials Science and Engineering Commons

Barwick, Brett E.; Gronniger, Glen; Yuan, Lu; Liou, Sy-Hwang; and Batelaan, Herman, "A measurement of electron-wall interactions using transmission diffraction from nanofabricated gratings" (2006). Faculty Publications: Materials Research Science and Engineering Center. 66.

https://digitalcommons.unl.edu/mrsecfacpubs/66

This Article is brought to you for free and open access by the Materials Research Science and Engineering Center at DigitalCommons@University of Nebraska - Lincoln. It has been accepted for inclusion in Faculty Publications: Materials Research Science and Engineering Center by an authorized administrator of DigitalCommons@University of Nebraska - Lincoln. 


\title{
A measurement of electron-wall interactions using transmission diffraction from nanofabricated gratings
}

\author{
Brett Barwick, Glen Gronniger, Lu Yuan, Sy-Hwang Liou, and Herman Batelaan ${ }^{a)}$ \\ Department of Physics and Astronomy, University of Nebraska-Lincoln, 116 Brace Laboratory, \\ P.O. Box 880111, Lincoln, Nebraska 68588-0111
}

(Received 11 March 2006; accepted 21 July 2006; published online 13 October 2006)

\begin{abstract}
Electron diffraction from metal coated freestanding nanofabricated gratings is presented, with a quantitative path integral analysis of the electron-grating interactions. Electron diffraction out to the 20th order was observed indicating the high quality of our nanofabricated gratings. The electron beam is collimated to its diffraction limit with ion-milled material slits. Our path integral analysis is first tested against single slit electron diffraction, and then further expanded with the same theoretical approach to describe grating diffraction. Rotation of the grating with respect to the incident electron beam varies the effective distance between the electron and grating bars. This allows the measurement of the image charge potential between the electron and the grating bars. Image charge potentials that were about $15 \%$ of the value for that of a pure electron-metal wall interaction were found. We varied the electron energy from 50 to $900 \mathrm{eV}$. The interaction time is of the order of typical metal image charge response times and in principle allows the investigation of image charge formation. In addition to the image charge interaction there is a dephasing process reducing the transverse coherence length of the electron wave. The dephasing process causes broadening of the diffraction peaks and is consistent with a model that ascribes the dephasing process to microscopic contact potentials. Surface structures with length scales of about $200 \mathrm{~nm}$ observed with a scanning tunneling microscope, and dephasing interaction strength typical of contact potentials of $0.35 \mathrm{eV}$ support this claim. Such a dephasing model motivated the investigation of different metallic coatings, in particular $\mathrm{Ni}, \mathrm{Ti}, \mathrm{Al}$, and different thickness $\mathrm{Au}-\mathrm{Pd}$ coatings. Improved quality of diffraction patterns was found for Ni. This coating made electron diffraction possible at energies as low as $50 \mathrm{eV}$. This energy was limited by our electron gun design. These results are particularly relevant for the use of these gratings as coherent beam splitters in low energy electron interferometry. (C) 2006 American Institute of Physics. [DOI: 10.1063/1.2357000]
\end{abstract}

\section{INTRODUCTION}

Coherent beam splitters for charged particles are needed to construct electron and ion interferometers. The most commonly used beam splitter to create a charged particle interferometer is the combination of a field emission tip (FET) and a biprism. This type of electron interferometer is often called a Möllenstedt-Düker interferometer and was demonstrated in $1955 .{ }^{1}$ FET biprism interferometers have been used to study several interesting effects, such as electron antibunching, ${ }^{2}$ the Aharonov-Bohm (AB) effect, ${ }^{3-5}$ the Sagnac effect, ${ }^{6}$ and decoherence due to an image charge dragging over a surface. ${ }^{7}$

While the FET biprism was ideally suited for observing many of the before mentioned effects, there are drawbacks to using the FET biprism as a coherent beam splitter. The FET interferometer is operated at ultrahigh vacuum pressures $\left(10^{-10}\right.$ Torr), which causes difficulties when used in studies that involve gases. Such experiments are determining the electron forward-scattering amplitude ${ }^{8}$ or collisional decoherence due to electron-gas collisions. ${ }^{9,10}$ These studies have not been done yet and would be more easily attained if a thermionic electron source could be implemented.

While the nondispersive nature of the scalar $\mathrm{AB}$ effect

${ }^{a)}$ Electronic mail: hbatelaan2@unl.edu with neutrons has been shown, currently the fundamental test to show the nondispersive nature of the vector $\mathrm{AB}$ for electrons has not yet been done. ${ }^{11,12}$ This crucial test requires low energy electron interferometry. The lowest energy interferometer demonstrated works at about $200 \mathrm{eV}{ }^{13}$ To reach lower energies both a grating interferometer and biprism interferometer are interesting to explore. These methods are complementary in that a biprism splits the electron wave front in coordinate space, while a grating splits the electron amplitude in momentum space. In practice this means that a grating yields well defined separate beams.

Another effect that has not yet been realized is the interferometric test of the mass shift of an electron between two conducting plates where it may be possible to see quantum electrodynamics (QED) corrections to the classical theory. ${ }^{14}$ All of the before mentioned experiments need an interferometer for electrons which in turn requires a charged particle coherent beam splitter. A thermionic electron source in conjunction with transmission gratings is the essential component for the construction of a low energy Mach-Zehnder electron interferometer which would allow the realization of the before mentioned experiments.

One method in which to make a charged particle coherent beam splitter is to employ diffraction. Electron diffraction at higher energies is a very well developed technique 
that began when Davisson and Germer, in their famous 1927 experiment, diffracted $70 \mathrm{keV}$ electrons from a periodic crystal structure. ${ }^{15}$ The Davisson and Germer experiment was based on reflective diffraction, which is used extensively today to study surface structures as with reflective high energy electron diffraction (RHEED) and low energy electron diffraction (LEED). Transmission electron diffraction was used by Marton et al. ${ }^{16,17}$ as a coherent beam splitter in an electron intereferometer. They used copper crystals with a periodicity on the order of $150 \AA$, and were able to construct a unique Mach-Zehnder-type interferometer with three of these crystals. The three crystal grating interferometer was operated at $60 \mathrm{keV}$ and suffered from low electron intensities, secondary electrons, and inelastic scattering in the crystals. These problems prevented this technology from seeing applications. The Möllenstedt-Düker approach quickly overtook the use of crystals. It is perhaps interesting to point out that although the grating interferometer approach has until now not played a role in electron interferometry it is one of the standard approaches in neutron, atom, and molecular interferometry.

Thin collodion films with gold strips deposited on them have been used for electron diffraction at $50 \mathrm{keV}$ by Holl in 1969. ${ }^{18}$ Much more recently Ito et al. have constructed beautiful nanofabricated Fresnel lenses and have used these in a $200 \mathrm{keV}$ conventional transmission electron microscope (CTEM) ${ }^{19}$ The Fresnel lenses are phase gratings that have been constructed by drilling holes with an electron beam in a substrate of $\mathrm{AlF}_{3}$. The problem of constructing a beam splitter for electrons using either periodic crystal structures, thin films with strip coatings, or phase Fresnel lenses is that these methods require fairly high electron energies for them to transmit electrons and as the electron energy is lowered inelastic scattering becomes more pronounced. At energies below $1 \mathrm{kV}$ the secondary electron emission and inelastic scattering through $100 \mathrm{~nm}$ membranes effectively stop due to the short penetration depth. ${ }^{20}$

Thus, for a low energy coherent beam splitter another approach is needed. A pure transmission grating or the Kapitza-Dirac effect can be used to diffract electrons. The latter was recently demonstrated by our group, but suffers from the fact that high laser intensities are needed, which currently can only be reached by pulsed lasers. ${ }^{21}$ By employing pulsed lasers the electron counts per second in the diffraction pattern are on the order of 1, making the construction of an electron interferometer employing the KapitzaDirac effect difficult. Pure transmission gratings for electrons were demonstrated in 1961 by Jönsson. ${ }^{22}$ Jönsson showed $50 \mathrm{keV}$ electron diffraction from single and multiple slits, which were made by pealing away a metal periodic structure that had been deposited on a glass substrate. The small number of slits limited the amount of throughput, and the irregularities of the slits limited the spatial quality of the electron wave front. Electron interferometry was never attempted with these gratings.

More recently high quality nanofabricated $100 \mathrm{~nm}$ periodicity transmission gratings have been made by Savas et al., ${ }^{23}$ and used extensively by Chapman et al. ${ }^{24}$ Grisenti et al., ${ }^{25}$ and Arndt et al. ${ }^{26}$ for both diffraction and interfer-

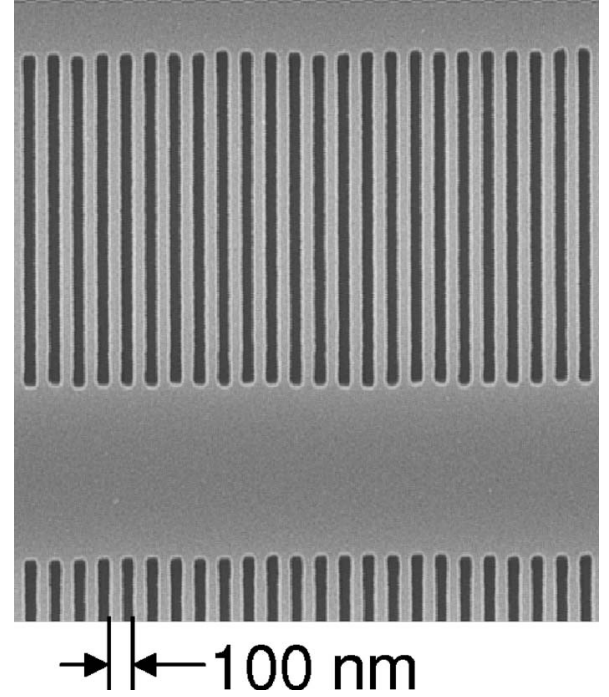

FIG. 1. Nanofabricated $100 \mathrm{~nm}$ period grating. Figure courtesy of Tim Savas.

ometry with atoms and molecules. The $100 \mathrm{~nm}$ periodicity gratings are produced using ultraviolet (UV) lithography in $\mathrm{Si}_{3} \mathrm{~N}_{4}$ substrates and a picture of a typical grating can be seen in Fig. $1 .^{23}$ These gratings with an added metallic coating have been shown by us in a dedicated apparatus to give high quality electron diffraction down to $125 \mathrm{eV}$ and Cronin and co-workers have shown $500 \mathrm{eV}$ diffraction from these gratings in a commercially available scanning electron microscope. $^{20,27}$

In this paper we present a detailed study of electron diffraction from these $100 \mathrm{~nm}$ periodicity metallic coated gratings. Even in the absence of secondary electron emission and inelastic scattering it is still possible that electron-grating interactions cause the electron wave to dephase. The usefulness of the gratings for low energy electron interferometry depends on the detailed nature and strength of these interactions. This is why we carefully measured the interactions. To understand the electron-grating interactions we developed a path integral model that agrees well with our experimental data. Other works have investigated both experimentally and theoretically the image charge potential on electrons and ions, with respect to different experimental situations including inelastic processes in a scanning transmission electron microscope (STEM), ion surface scattering, and elastically scattered electrons in LEED and RHEED experiments. ${ }^{28-31}$

High quality single slits were developed using a focused ion beam (FIB) milling machine to allow better collimation of our electron beams. We show that these high quality single slits produce a diffraction limited beam which allowed us to examine the electron-grating interactions more accurately.

In this study we investigate two potentials by studying far field electron diffraction patterns. The image charge potential strength is determined for energies of 100 and $900 \mathrm{eV}$. In the course of our previous qualitative study an unexpected broadening of the diffraction peaks was observed. ${ }^{27}$ We show that the broadening of the diffraction peaks is affected by the type and thickness of metallic coating, and we model this broadening by introducing a random 
potential into our diffraction model. We present data at $900 \mathrm{eV}$ that show diffraction out to the 20th diffraction order, which attests to the quality of the gratings, and finally we show electron diffraction at $50 \mathrm{eV}$ that leads us to believe that these gratings may be used to make a low energy electron interferometer.

\section{THEORY}

\section{A. Theory of single slit electron diffraction}

To model single slit electron diffraction we use the Fraunhofer limit of Kirchoff's optical diffraction theory. Kirchoff's optical diffraction theory or scalar diffraction theory is a solution to the Helmholtz equation,

$$
\left[\nabla^{2}+k(\mathbf{x})^{2}\right] \phi_{k}(\mathbf{x})=0 .
$$

It can be seen that Kirchhoff's optical diffraction theory can be used for matter waves, $\phi_{k}(\mathbf{x})$, by identifying the wave number $k(\mathbf{x})$ as

$$
k(\mathbf{x})=\sqrt{2 m[E-V(\mathbf{x})] / \hbar^{2}},
$$

where $k=\left.\sqrt{2 m E / \hbar^{2}}\right|_{V=0} \cdot 32$

This approach holds for time-independent potentials and scalar wave functions. ${ }^{32}$

For the case of a one-dimensional (1D) single slit, illuminated by a plane wave, the familiar single slit diffraction solution for the probability distribution $I(x)$ is obtained when Eq. (1) is solved in the Fraunhofer limit,

$$
I(x)=I(0)\left[\frac{\sin (\beta)}{\beta}\right]^{2},
$$

where $\beta=k w x / \sqrt{L^{2}+x^{2}}$, with $x$ the position on the detection screen, $L$ the distance between the slit and screen, $k$ the wave number, and $w$ the slit width. ${ }^{33}$ This solution is compared to our experimental data and the path integral results in Figs. 5 and 6. This idealized approach to single slit diffraction is not completely realized in our experiment. First, we do not have perfect plane wave illumination of our single slit. Second, we have limited resolution in our detection plane due to the finite size of our detection slit, and third, a $1 / r$ dependent image charge potential modifies this pattern. ${ }^{34}$ We would like the agreement to be as good as possible, because our goal is to understand and measure electron-wall interactions. To get better agreement between experiment and theory, and have a model that is flexible for different grating geometries, we use a modified form of Feynman's path integral formalism that he presented in his paper, Space-Time Approach to NonRelativistic Quantum Mechanics published in Review of Modern Physics in $1948 .{ }^{35}$ This time-independent path integral method that sums over only the classical straight line paths is very similar to that of Turchette. ${ }^{36}$ By using the path integral method to model electron diffraction, we can implement an incoherent source and convolute over a detection slit, and at the same time predict near and far field patterns without changing any equations. We should mention at this point that no incoherent summation over electron velocities is needed given our small electron energy spread (see Sec. III).

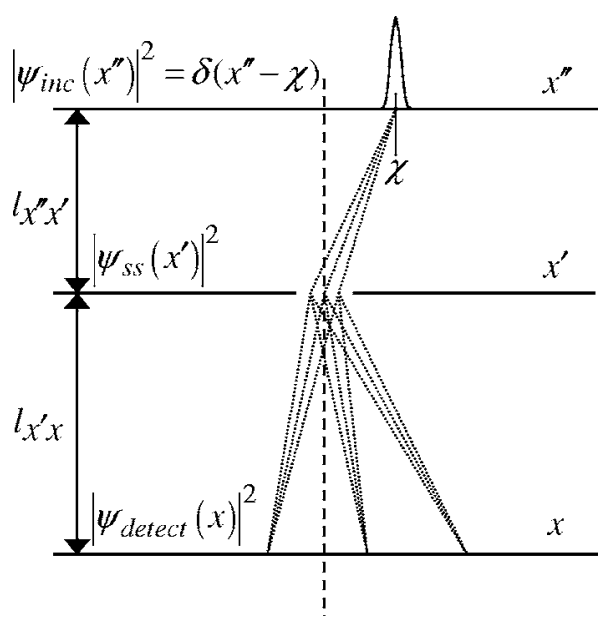

FIG. 2. Geometry of the single slit theory. There are three axes, with $x^{\prime \prime}$ depicting the incoherent slit plane, $x^{\prime}$ depicting the single slit plane, and $x$ depicting the detection plane. The distances between the planes are $l_{x^{\prime \prime} x^{\prime}}$ and $l_{x^{\prime} x}$. The dashed line is the zero point for the $x^{\prime \prime}, x^{\prime}$, and $x$ variables. The dotted lines show a few of the possible straight paths that the electron can take. All phases that are accumulated along these paths are stored in the kernel. The position $\chi$ of the initial delta function indicates the location of the incoherent source.

We can consider the first collimation slit to be the incoherent source. To describe this incoherent source we start with the wave function,

$$
\Psi_{\text {inc }}\left(x^{\prime \prime}, \chi\right)=\delta\left(x^{\prime \prime}-\chi\right)
$$

where $x^{\prime \prime}$ is a position on the incoherent source plane, with a point source at position $\chi$ (see Fig. 2). We will later incoherently integrate over the $\chi$ position. The next step is to find the wave function on the single slit plane, $\Psi\left(x^{\prime}, \chi\right)$. This is done by propagating $\Psi_{\text {inc }}\left(x^{\prime \prime}, \chi\right)$ using the Kernel $K_{x^{\prime \prime} \rightarrow x^{\prime}}\left(x^{\prime \prime}, x^{\prime}\right)$,

$$
\Psi\left(x^{\prime}, \chi\right)=\int_{-\infty}^{\infty} K_{x^{\prime \prime} \rightarrow x^{\prime}}\left(x^{\prime \prime}, x^{\prime}\right) \Psi_{\mathrm{inc}}\left(x^{\prime \prime}, \chi\right) d x^{\prime \prime}
$$

where the Kernel is defined as

$$
K_{x^{\prime \prime} \rightarrow x^{\prime}}\left(x^{\prime \prime}, x^{\prime}\right)=\exp \left[i 2 \pi \sqrt{\left(x^{\prime \prime}-x^{\prime}\right)^{2}+\left(l_{x^{\prime \prime} x^{\prime}}\right)^{2}} / \lambda_{d B}\right]
$$

with $x^{\prime}$ being a position on the single slit plane, and $l_{x^{\prime \prime} x^{\prime}}$ the distance between the incoherent slit and the single slit plane. The form of the Kernel in Eq. (6) will be reused with different $x^{i}$ variables corresponding to different $l_{x^{i} x}$, which are the distances between different planes.

To construct a slit we introduce the function

$$
\Pi_{w_{i}}\left(x^{\prime}\right)=H\left(x^{\prime}+w_{i} / 2\right) H\left(-x^{\prime}+w_{i} / 2\right),
$$

which is defined by using two Heaviside functions, with $w_{i}$ being the width of the slit. Additionally, a phase shift due to an image charge potential must be added. To model this potential we treat each side of the single slit as an infinite plane. The electric potential due to an induced image charge in each wall is 


$$
V_{\text {image }}\left(x^{\prime}\right)=\frac{q_{\text {image }}}{4 \pi \varepsilon_{0}}\left[\frac{1}{\left(w_{\mathrm{ss}}+2 x^{\prime}\right)}+\frac{1}{\left(w_{\mathrm{ss}}-2 x^{\prime}\right)}\right]
$$

where $w_{\mathrm{ss}}$ is the width of the single slit, and $q_{\mathrm{image}}$ is a parameter that allows us to adjust the strength of the image charge potential. The phase accumulated by an electron that goes through this potential is

$$
\varphi_{\text {image }}\left(x^{\prime}\right)=\frac{-q V_{\text {image }}\left(x^{\prime}\right) t}{\hbar},
$$

where $q$ is the charge of an electron $(-e)$, and $t=l / \nu_{e}$, which is the time it takes for the electron to travel through the slit, $l$ is the substrate thickness, and $\nu_{e}$ is the electron velocity. We only consider straight paths through the potential. This is a very good approximation due to the weakness of the potential combined with the short interaction time. The wave function after the electron passes through the single slit is

$$
\Psi_{\mathrm{ss}}\left(x^{\prime}, \chi\right)=\Psi\left(x^{\prime}, \chi\right) \Pi_{w_{\mathrm{ss}}}\left(x^{\prime}\right) e^{i \varphi_{\text {image }}\left(x^{\prime}\right)} .
$$

Now the final wave function on the detection screen can be found by propagating $\Psi_{\mathrm{ss}}\left(x^{\prime}, \chi\right)$ to the detection screen,

$$
\Psi_{\text {detect }}(x, \chi)=\int_{-\infty}^{\infty} K_{x^{\prime} \rightarrow x}\left(x^{\prime}, x\right) \Psi_{\mathrm{ss}}\left(x^{\prime}, \chi\right) d x^{\prime},
$$

with $x$ being a position on the detection plane and where $l_{x^{\prime} x}$ is the distance between the single slit and the detection plane and $K_{x^{\prime} \rightarrow x}\left(x^{\prime}, x\right)$ is the propagator from the single slit to the detection plane. The probability on the detection plane is

$$
P_{\text {detect }}(x, \chi)=\left|\Psi_{\text {detect }}(x, \chi)\right|^{2},
$$

which is the diffraction pattern formed by one of the delta function point sources located on the incoherent source plane. To obtain a probability that can be compared to experimental data we convolute $P_{\text {detect }}(x, \chi)$ with the detection slit and integrate $\chi$ over the incoherent slit,

$$
P_{\text {final }}(x)=\Pi_{w_{3}}(x) * \int_{-w_{1} / 2}^{+w_{1} / 2} P_{\text {detect }}(x, \chi) d \chi,
$$

where the symbol $*$ denotes a convolution, $w_{1}$ is the width of the first slit, and $w_{3}$ is the width of the detection slit. Finally $P_{\text {final }}(x)$ can be normalized to the experimental data and compared.

To obtain $P_{\text {final }}(x)$ we evaluated the integrals numerically by replacing all integrations with summations. The step sizes for each of the planes were chosen such that each adjacent point had a phase difference much less than $2 \pi$. Typical step sizes on each plane were $\sim 2 \mathrm{~nm}$ for $900 \mathrm{eV}$ electrons and $\sim 0.5 \mathrm{~nm}$ for $100 \mathrm{eV}$ electrons.

\section{B. Theory of multiple slit electron diffraction}

Diffraction of a plane wave by a multislit grating in the Fraunhofer limit has an analytic solution for the probability which is given by

$$
I(x)=\frac{I(0)}{N^{2}}\left[\frac{\sin (\beta)}{\beta}\right]^{2}\left[\frac{\sin (N \alpha)}{\sin (\alpha)}\right]^{2},
$$

where $\beta$ is the same as defined in the previous section (with $w$ given by the grating windows) and where $\alpha$ $=k d x / \sqrt{L^{2}+x^{2}}$, with $x$ the position on the detection screen, $L$ the distance between the slit and screen, $k$ the propagation number, $N$ the number of slits, and $d$ the periodicity of the grating. ${ }^{33}$ This analytic solution does not accurately represent our experimental parameters, for the same reasons as mentioned above for the single slit solution. However, this analytic solution is useful to estimate the resolving power of a diffraction pattern,

$$
R \equiv \frac{d_{\text {peak }}}{w_{\text {peak }}}=\left.\frac{N d\left|\sin \theta_{\text {diff }}-\sin \theta_{0}\right|}{\lambda_{d B}}\right|_{\theta_{0}=0} \simeq N,
$$

with $d_{\text {peak }}$ the diffraction peak separation, $w_{\text {peak }}$ the diffraction peak width, $\theta_{\mathrm{diff}}$ is the diffraction angle, and $\theta_{0}$ the incident beam angle. ${ }^{33}$ This means that the transverse coherence length $l_{t}$ can be estimated from the observed diffraction pattern, $l_{t}=R d$.

To develop a more complete model for electron diffraction from metal coated nanofabricated gratings, we follow the approach described above. Another plane where the grating is located must be inserted in between the single slit and the detection plane. The new wave function located on the grating plane is

$$
\Psi\left(x^{g}, \chi\right)=\int_{-\infty}^{\infty} K_{x^{\prime} \rightarrow x^{g}}\left(x^{\prime}, x^{g}\right) \Psi\left(x^{\prime}, \chi\right) d x^{\prime},
$$

which includes a new variable $x^{g}$ for the grating plane, and also uses a new kernel, $K_{x^{\prime} \rightarrow x^{g}}\left(x^{\prime}, x^{g}\right)$, and has $l_{x^{\prime} x^{g}}$ as the distance between the single slit plane and the new grating plane.

To describe the grating we introduce the function

$$
\operatorname{comb}\left(\frac{x^{g}}{d}\right)=\sum_{n=-\infty}^{\infty} \delta\left(\frac{x^{g}}{d}-n\right)
$$

which is an infinite series of delta functions that are spaced by $d$ the periodicity of the grating. ${ }^{37}$ To set up a grating function that includes the individual slit widths of the grating, and the associated image charge phase shift in each slit we define

$$
\begin{aligned}
& G\left(x^{g}\right)=\left[S_{\text {diff }}\left(x^{g}\right) * \operatorname{comb}\left(\frac{x^{g}}{d}\right)\right] e^{i \varphi_{\text {rand }}\left(x^{g}\right)}, \\
& S_{\text {diff }}\left(x^{g}\right)=\Pi\left(x^{g}\right) e^{i \varphi_{g s}\left(x^{g}\right)},
\end{aligned}
$$

where the convolution of a single slit function $S_{\text {diff }}\left(x^{g}\right)$ with the comb function, and a phase shift $\varphi_{\text {rand }}\left(x^{g}\right)$ due to a random potential are used to construct the grating. The function $S_{\text {diff }}\left(x^{g}\right)$, which includes a rectangular function $\Pi\left(x^{g}\right)$ and a phase shift $\varphi_{g s}\left(x^{g}\right)$ due to the image charge potential, will be defined explicitly in the next sections. The random phase shift that causes broadening of the diffraction peaks, described by $\varphi_{\text {rand }}\left(x^{g}\right)$, will also be describe in a later section. 
The wave function of the electron after it has gone through the grating is thus

$$
\Psi_{\text {grating }}\left(x^{g}, \chi\right)=\Psi\left(x^{g}, \chi\right) G\left(x^{g}\right) .
$$

The wave function $\Psi_{\text {grating }}\left(x^{g}, \chi\right)$ is propagated to the detection plane with the kernel, $K_{x^{g} \rightarrow x}\left(x^{g}, x\right)$, with $l_{x^{g} x}$ being the distance between the grating and detection plane. The wave function on the detection plane is

$$
\Psi_{\text {detect }}(x, \chi)=\int_{-\infty}^{\infty} K_{x^{g} \rightarrow x}\left(x^{g}, x\right) \Psi_{\text {grating }}\left(x^{g}, \chi\right) d x^{g} .
$$

To find the probability on the detection screen this $\Psi_{\text {detect }}(x, \chi)$ can be substituted in Eqs. (12) and (13) to give a result that can be compared to our data.

\section{Image charge potential}

When an electron passes through the grating its interaction with the grating bars can be described with an image charge potential. If we treat the grating bar walls as infinite planes, we can use the well known image charge solution to Laplace's equation. This method of using an infinite plane to describe the interaction between the particle and the grating bar wall has been used before to describe the van der Waals interaction between atoms (or molecules) and the trapezoidal shaped bar of the nanofabricated gratings. ${ }^{38-40}$ In Sec. II A, we included an image charge potential; however, that potential did not take into account the geometry of the walls. We take this geometry into account when describing the interaction between the electron and the grating bar walls.

As can be seen in Fig. 3, the distance between the electron and both the left and right walls must be known to calculate the image potential. The distance from the left (or right) wall to a point in between the walls is

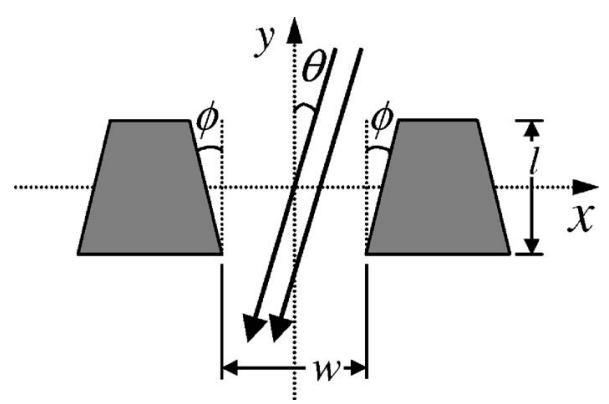

FIG. 3. Cross sectional cut of the nanofabricated grating. The bevel angle of the slit is given by $\phi$, the electron beam angle with respect to the grating is $\theta$, the slit width is given as $w$, and the grating thickness is given by $l$.

$$
d_{\text {left/right }}=\frac{ \pm x+y \tan (\phi)+w / 2+l / 2 \tan (\phi)}{\sqrt{1+\tan ^{2}(\phi)}},
$$

where $\phi$ is the bevel angle of the wall, $l$ is the substrate thickness, and $w$ is the slit width. Knowing the distance from a point to each wall allows us to set up the induced electric potential due to the image charge,

$$
V_{\text {tot }}(x, y)=V_{\text {left }}(x, y)+V_{\text {right }}(x, y),
$$

where

$$
V_{\text {left/right }}(x, y)=\frac{q_{\text {eff }}}{8 \pi \varepsilon_{0} d_{\text {left/right }}},
$$

with $q_{\text {eff }}$ representing the image charge. We use $q_{\text {eff }}$ to adjust the strength of the image charge potential, which is related to the permittivity of the grating material as follows: $q_{\text {eff }}$ $=+e\left(\varepsilon-\varepsilon_{0}\right) /\left(\varepsilon+\varepsilon_{0}\right)$. The phase shift accumulated by the electron is

$$
\phi_{\text {left/right }}(x)=-\frac{q}{\nu_{e} \hbar} \int_{l / 2}^{-l / 2} V_{\text {left/right }}(x, y) d y,
$$

with $q$ representing the charge of an electron $(-e)$. If the integration is carried out, the explicit equation for $\phi_{\text {left/right }}(x)$ is given by

$$
\phi_{\text {leftright }}(x)=\left.\frac{-q_{\mathrm{eff}} q \sqrt{\tan ^{2} \phi+1} \ln (y \tan \phi \pm x+w / 2+l / 2 \tan \phi)}{8 \pi \varepsilon_{0} \nu_{e} \hbar \tan \phi}\right|_{y=l / 2} ^{y=-l / 2} .
$$

The effective charge $q_{\text {eff }}$ is expected to be less than the electron charge. The first reduction in the effective charge is due to successive reflective image charges. These occur when an image charge causes another image charge to form in the opposite wall. While this does not change the form of the potential it does add a factor of $\ln (2)$, when all of these successive image charges are taken into account for the case of parallel walls. ${ }^{14}$ The second factor that reduces $q_{\text {eff }}$ is that we likely do not have a metallic coating on the walls inside each slit. Instead the image charge is formed in the dielectric. The solution for an image charge in a dielectric has an addi- tional multiplicative factor of $\chi_{e} /\left(\chi_{e}+2\right) \simeq 0.8$, with $\chi_{e}=8$

being the value of the electronic susceptibility of $\mathrm{Si}_{3} \mathrm{~N}_{4} \cdot{ }^{41,42}$ Also the geometry of the slit may cause another reduction in the image charge strength, because the walls are not infinite planes.

In our model we only consider straight line paths through the slit so this excludes much weaker quantum mechanical corrections such as bound states between an electron and its image charge, known as "murium." ignore a process proposed by Anglin and Zurek; an image charge will experience Ohmic resistance in the material it 
was formed in, causing decoherence. ${ }^{44}$ While these twoeffects are expected to be small at our current electron energies, at lower energies they may become important.

\section{Random potential}

One unexpected aspect of our measured diffraction patterns was that the peaks seemed to be significantly wider than that of initial beam. This broadening of the diffraction peaks is caused by the grating. We want to find a model that mimics this broadening. The fact that the transverse coherence length determines the width of the diffraction peaks motivates our model (see Sec. II B). If the grating reduces the effective transverse coherence length broadening is obtained. The reduction in coherence length can be modeled by a series of random static Gaussian potentials. The characteristic width of the coherence length needed is about $300 \mathrm{~nm}$ to obtain the observed diffraction peak width. This means that the lateral width of the Gaussian potentials must be of the same order. In our random potential model we choose to have a static potential of the form

$$
V_{\text {rand }}(x)=\sum_{i} A_{i} e^{-4 \ln 2\left(x-x_{i}\right)^{2} / \sigma_{i}^{2}} .
$$

The parameters $\sigma_{i}$ and $A_{i}$ are obtained from random number generators. The set of $\left\{x_{i}\right\}$ of centers is regularly spaced and separated by $2 \sigma_{i}$. The random number generators are Gaussian distributions with means of $250 \mathrm{~nm}$ for $\sigma_{i}$ and $0 \mathrm{eV}$ for $A_{i}$. The full width at half maximum (FWHM) is $250 \mathrm{~nm}$ and $0.35 \mathrm{eV}$ for each of the random number generators, respectively. The amplitudes $A_{i}$ give a phase shift on the order of $\pi$ for values of $0.2 \mathrm{eV}$. For smaller values no broadening occurs. Each realization of the random potential leads to a different diffraction pattern. We sum incoherently over these patterns to obtain our final calculated result. This sum is motivated by the fact that electrons going through different heights of the grating contribute incoherently to the diffraction pattern (see Sec. III). These electrons pass through different portions of the grating and are thus expected to "see" a different potential. When this random potential is added to the grating function, it indeed broadens the individual peaks. Also the phase shift accumulated when an electron passes through this potential scales linearly with the electron velocity.

The physical system that motivates the above model is based on contact potentials. The typical size of contact potentials is $0.1-1 \mathrm{~V}$, and agrees with the value needed for the potential amplitudes. For example, the contact potential between different crystal faces of copper is about $0.4 \mathrm{~V} .{ }^{45}$

The use of time-independent conservative potentials within the context of a path integral formulation also means that the electron energy is not modified by the potentials. This is why we do not discuss the longitudinal coherence length in this context.

\section{E. Grating tilt dependence of potentials}

Because the random potential has increased the number of fit parameters it is useful to also increase the number of experimental parameters. An important example of such a parameter is the grating tilt angle $\theta$ (see Fig. 3). Tilting the grating allows us to effectively vary the distance between the electron and the grating bar walls. Doing these grating tilt experiments allows us to characterize both the physical structure of the grating (bevel angle, thickness, and individual slit width) and how those physical parameters and the image charge potential affect diffraction patterns in the far field. This method has been used before by Cronin and Perreault to obtain a value for the van der Waals interaction coefficient $C_{3}$, between sodium atoms and a grating made of $\mathrm{Si}_{3} \mathrm{~N}_{4} \cdot{ }^{34}$ The use of a tilted grating has also been recently used to determine the size of the helium trimer. ${ }^{46}$

To model the tilting of the grating and the subsequent far field patterns, we substitute

$$
x=y \tan \theta+x^{g}
$$

into Eq. (22). The parameter $\theta$ is the angle between the electron beam axis and the grating. The $x$ coordinate is in the plane of the grating for $\theta=0$, and the $x^{g}$ coordinate is in the plane of the grating for arbitrary $\theta$. When the integration over $y$ is carried out, the result is

$$
\phi_{\text {leftright }}\left(x^{g}\right)=\left.\frac{-q_{\mathrm{eff}} q \sqrt{\tan ^{2} \phi+1} \ln \left[y(\tan \phi \pm \tan \theta) \pm x^{g}+w / 2+l / 2 \tan \phi\right]}{8 \pi \varepsilon_{0} \nu_{e} \hbar(\tan \phi \pm \tan \theta)}\right|_{y=l / 2} ^{y=-l / 2} .
$$

The full phase shift accumulated by traveling straight between two grating bars is

$$
\phi_{g s}\left(x^{g}\right)=\phi_{\text {left }}\left(x^{g}\right)+\phi_{\text {right }}\left(x^{g}\right),
$$

The phase $\phi_{g s}\left(x^{g}\right)$ can be inserted into the grating function [Eq. (26)] mentioned above to predict an electron diffraction pattern. Or vice versa, the diffraction pattern provides information on the strength and spatial dependence of the image charge potential.

As the tilt angle increases the opening between the grating bars closes. We define the center of the opening between two bars as the zero of the $x$ coordinate, while the left bar edge is indicated with $w_{\text {left }}$ and the right bar edge is indicated with $w_{\text {right }}$ in the same coordinate system. The limiting angle for which the opening has completely closed is 


$$
\theta_{\max }=\tan ^{-1}\left(\frac{w+l \tan \phi}{l}\right) .
$$

The values of $w_{\text {left }}$ and $w_{\text {right }}$ are used to construct the $\Pi\left(x^{g}\right)$ function used in Eq. (19), which is defined as

$$
\Pi\left(x^{g}\right)=H\left(x^{g}+w_{\text {left }} / 2\right) H\left(-x^{g}+w_{\text {right }} / 2\right) .
$$

The edge positions of each slit vary as a function of $\theta$ and are given by

$$
\begin{aligned}
& w_{\text {left }}=-w / 2+l / 2 \tan \theta, \quad \theta<\phi, \\
& w_{\text {left }}=-w / 2+l / 2 \tan \theta, \quad \theta>\phi, \\
& w_{\text {left }}=-w / 2-l / 2 \tan (\theta)-l \tan \phi, \quad \theta<-\phi, \\
& w_{\text {right }}=w / 2+l / 2 \tan \theta, \quad \theta<\phi, \\
& w_{\text {right }}=w / 2+l / 2 \tan \theta-l \tan \phi, \quad \theta>\phi, \\
& w_{\text {right }}=w / 2-l / 2 \tan (\theta), \quad \theta<-\phi,
\end{aligned}
$$

and are a function of the slit width $w$, grating thickness $l$, and bevel angle $\phi$ of the grating bars.

Another parameter that changes as a function of grating tilt $\theta$ is the effective periodicity of the grating. To account for this in our model, the periodicity $d$ of the grating is changed to

$$
d_{\mathrm{eff}}=d \cos \theta,
$$

in Eq. (17), where $d_{\text {eff }}$ is the effective periodicity of the tilted grating.

The random potential model must also be modified to account for grating tilt. It is assumed that the potentials are essentially one dimensional. To model the tilt in the random potential we use substitute $x=x^{g} / \cos (\theta)$ into Eq. (27) and define the random phase as

$$
\phi_{\text {rand }}\left(x^{g}\right)=\frac{-V_{\text {rand }}\left(x^{g}\right) l}{\nu_{e} \hbar},
$$

which can now be inserted into Eq. (25).

\section{EXPERIMENTAL SETUP}

The experiment was done in a stainless steel vacuum chamber at $10^{-7}$ Torr, which has two layers of magnetic shielding. The magnetic fields inside the shielding were on the order of $5 \mathrm{mG}$. The thermionic source used was a tungsten filament, with several electrostatic lenses to focus the emitted electrons. The electrons leave the gun with a beam size of approximately $1 \mathrm{~mm}$ in diameter.

A schematic of the experimental system, including all major components, is illustrated in Fig. 4. The electron beam is collimated by two slits, the first slit is $\sim 5 \mu \mathrm{m}$ wide $\times 3 \mathrm{~mm}$ tall and is located $2 \mathrm{~cm}$ from the end of the electron gun. The second slit is $2 \mu \mathrm{m}$ wide $\times 10 \mu \mathrm{m}$ tall and is located $23 \mathrm{~cm}$ from the first slit. After the electrons pass through the first two collimation slits they continue to the metal coated $\mathrm{Si}_{3} \mathrm{~N}_{4}$ grating located at a distance of $5.5 \mathrm{~cm}$ from the second slit. To make all three slits parallel the first and third slits are placed in Picomotor ${ }^{\mathrm{TM}}$ driven rotational

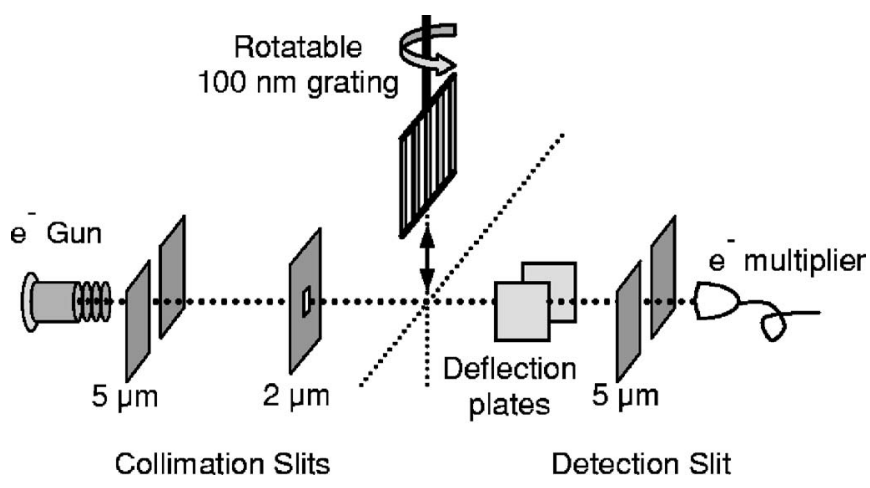

FIG. 4. Illustration of the experimental setup. The electrons are created in the electron gun using a tungsten filament. The filament is floated at a negative voltage and the electrons are focused using several lenses. After leaving the source the electrons are collimated by 5 and $2 \mu \mathrm{m}$ slits. After traveling through the two collimation slits the electrons pass through a metallic coated nanofabricated grating, which can be moved into the beam with a vertical linear feedthrough. After passing through the diffraction grating the diffraction pattern is scanned by either moving a $5 \mu \mathrm{m}$ detection slit across the pattern or by rastering the electron beam with deflection plates. The electrons are then detected with an electron multiplier placed behind the detection slit.

mounts. The detection slit (third slit) is $5 \mu \mathrm{m}$ wide and is located at a distance of $24 \mathrm{~cm}$ downstream from the grating. Both collimation slits and the detection slit are mounted on linear feedthroughs so that each can be centered around the electron beam. Several circular apertures, placed along the beam path, reduce background electron counts.

To obtain well collimated beams at the detection plane, slits were machined by a FIB in $100 \mathrm{~nm}$ thick silicon nitride membrane windows. The $100 \mathrm{~nm}$ membrane windows were first coated with $\sim 5 \mathrm{~nm}$ of Ti on both sides, then $\sim 10 \mathrm{~nm}$ of $\mathrm{Au}$ on both sides. The Ti coating was used so that the $\mathrm{Au}$ would better adhere to the substrate membrane. Different substrates were made with slits of $\sim 100 \mathrm{~nm}, 1 \mu \mathrm{m}$, and $2 \mu \mathrm{m}$, each with a height of $10 \mu \mathrm{m}$. The $\sim 100 \mathrm{~nm}$ slit is the most difficult to make because it is near to the resolution limit of the FIB machine. We choose to use the $2 \mu \mathrm{m}$ slit to collimate the beam for multislit diffraction, because the $100 \mathrm{~nm}$ and $1 \mu \mathrm{m}$ slits are diffraction limited, which increases the beam width (see Sec. IV A).

The collimation provided by the first two slits can be described classically by the angular spread of the subsequent electron beam. This angular spread is related to the geometrically allowed angles and is $\theta_{\text {geom }}=(5+2) \mu \mathrm{m} / 25 \mathrm{~cm}=2.8$ $\times 10^{-5} \mathrm{rad}^{27}$ This $\theta_{\text {geom }}$ assumes a $2 \mu \mathrm{m}$ second slit and a $5 \mu \mathrm{m}$ first slit. The geometric beam expected at the detection plane is approximately $8 \mu \mathrm{m}$; however, at $900 \mathrm{eV}$ we see a beam width of $\sim 16 \mu \mathrm{m}$. Part of the reason that the beam is wider than expected is that the second slit degrades over time. This degradation is likely due to contamination around the slit opening on the substrate surface. We have observed that $5 \mu \mathrm{m}$ laser machined slits after long electron beam exposure times become discolored and need cleaning with acid. This discoloration is likely due to residual oil in the vacuum that has charred onto the surface of the slit, and is therefore nonconductive. A repulsive potential was added to model how charging causes the second slit to act as a lens. In the path integral model without a grating the strength of the 
repulsive potential is adjusted to find agreement between the theoretical beams and the experimental beam.

Another aspect of the electron beam collimation is the longitudinal coherence. In our system the electron energy is set by floating the tungsten filament at a negative voltage. However, while this determines the electron energy there is an additional energy spread. Thermionic emission typically yields an energy spread, $\Delta E$ on the order of $1 \mathrm{eV}$. For the ranges of energies we use $(50-900 \mathrm{eV})$, this energy spread is negligible. To estimate how the energy spread can broaden an electron diffraction order, the diffraction angle, de Broglie wavelength, and electron energy are related in the following way:

$$
\Delta \theta_{\text {diff }} / \theta_{\text {diff }}=n \Delta \lambda_{\mathrm{dB}} / \lambda_{\mathrm{dB}}=n \Delta E / 2 E \approx n 10^{-3},
$$

where $n$ is the diffraction order. The associated longitudinal coherence length is about $50 \mathrm{~nm}$ using $l_{c}=h \sqrt{2 \bar{E} / m_{e}} / \Delta E$. The coherence time is $t_{c}=l_{c} / \nu_{e}$ and for our experiment is approximately $5 \times 10^{-15} \mathrm{~s}$.

The far field diffraction pattern is taken by moving the diffraction pattern across a $5 \mu \mathrm{m}$ slit at the detection plane by a set of electrostatic defection plates (Fig. 4). After the electrons pass through the detection slit they are detected with a Sjuts Model KBL510 channel electron multiplier. The deflection plates are separated by $2 \mathrm{~cm}$ and are composed of $\sim 5 \mathrm{~cm}$ square plates with the center located $14 \mathrm{~cm}$ from the diffraction grating, and $10 \mathrm{~cm}$ before the detection slit. To take data an SRS DS345 function generator is used to put a voltage on one of the deflection plates. The DS345 function generator is set to give a linear ramping voltage with a frequency of $0.1 \mathrm{~Hz}$, which starts at a negative voltage and ramps to a positive voltage. At the beginning of each ramping cycle a trigger pulse is sent to a mutichannel scalar board that is used to collect the pulses from the channel electron multiplier. The multichannel scalar board keeps track of the electron counts and stores them in bins corresponding to different times in the voltage ramp. The different time bins correspond directly to different positions on the detection plane. To calibrate the output of the multichannel scaling board to positions on the detection plane, a diffraction pattern is taken, then the third slit is moved a known amount (usually on the order of a few $100 \mu \mathrm{m}$ ), and another diffraction pattern is taken. Moving the third slit or scanning the deflection plates gives essentially identical patterns.

Typical scan times to collect a diffraction pattern are a few minutes. Longer times were needed to collect the diffraction patterns from the $100 \mathrm{~nm}$ single slit, because electron throughput is lower. To get diffraction patterns from the grating that show more than ten orders scan times of over an hour were needed.

\section{RESULTS}

The order in which the experiments are presented follows the ordering of the theory sections; first we discuss our single slit results, and second we discuss our grating results. The single slit results are considered to be a simple test case for the theory as compared to the increased complexity of the grating.

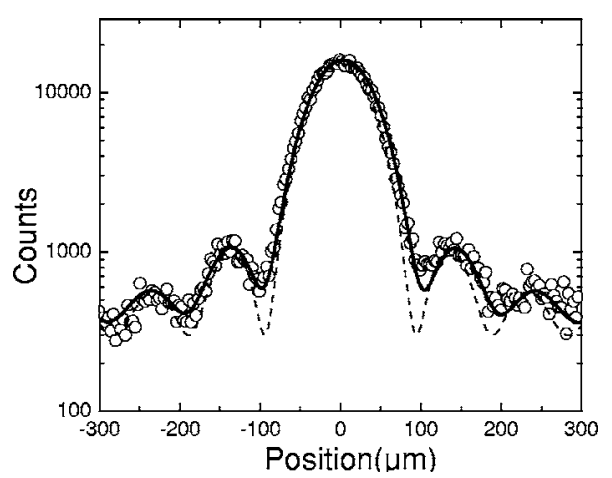

FIG. 5. $140 \mathrm{~nm}$ single slit diffraction, taken at $500 \mathrm{eV}$. The dots represent the experimental data, and the solid lines represent the results of the path integral model including incoherent averaging over the first slit and convolution over the detection slit. The dashed line represents the Fraunhofer optical diffraction theory with a plane wave illumination of the single slit. The theory is normalized to the experimental data.

\section{A. Single slit diffraction}

Experimentally the single slit is needed to collimate the electron beam, so that the diffraction peaks from the grating can be resolved. But without the grating the single slit can also give a diffraction pattern. Two limiting cases are straightforward. For wide slits the electron beam is defined geometrically without any visible diffraction. If the slit is narrowed the beam becomes better collimated up to the point where single slit diffraction starts to broaden the beam. There is thus an optimum slit width. ${ }^{47}$ Figures 5 and 6 show that slits narrower than $1 \mu \mathrm{m}$ increase the beam width. We find that at $2 \mu \mathrm{m}$ no diffraction is observed, and the best collimation is reached.

With $500 \mathrm{eV}$ electrons the single slit diffraction pattern can clearly be seen when the $\mathrm{Si}_{3} \mathrm{~N}_{4}$ substrate with a nominally $100 \mathrm{~nm}$ slit is inserted in the electron beam (see Fig. 5).

The theoretical curve plotted is the result of our path integral calculation with an incoherent first slit and a $140 \mathrm{~nm}$ single slit. The deviation in the slit width, $140 \mathrm{~nm}$ as opposed to $100 \mathrm{~nm}$, is expected because the nominal width was determined with the ion mill, run in the ion microscope mode. The resolution of the ion microscope was several tens of nanometer. The theoretical calculation also includes the

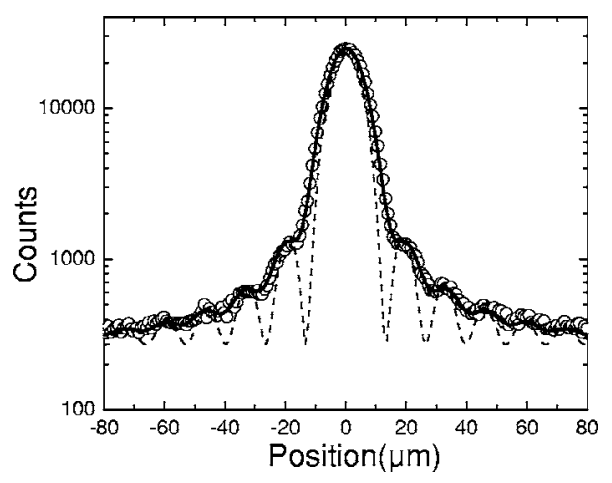

FIG. 6. $1 \mu \mathrm{m}$ single slit diffraction, taken at $500 \mathrm{eV}$. The dots represent the experimental data, and the solid lines represent the results of the path integral model including incoherent averaging over the first slit and convolution over the detection slit. The dashed line represents the Fraunhofer optical diffraction theory with a plane wave illumination of the single slit. The theory is normalized to the experimental data. 


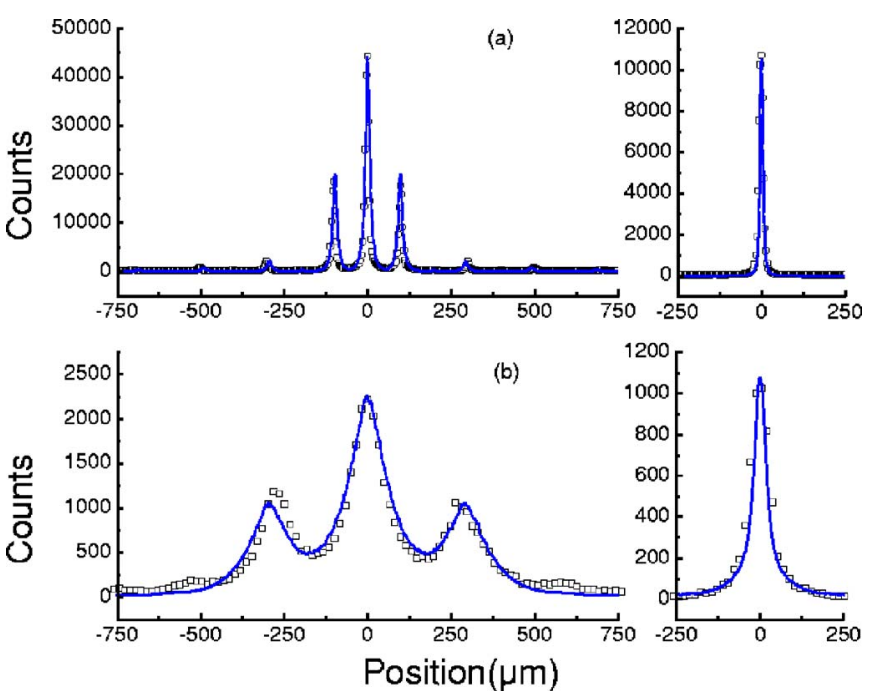

FIG. 7. (Color online) Electron diffraction (a) at 900 and (b) $100 \mathrm{eV}$. The diffraction patterns are on the left and the beam profiles with the gratings removed are on the right. The solid line is the path integral model with random potential, image charge, and incoherent source.

effect of the image charge potential. The agreement between experiment and theory is excellent. In the theoretical curve a background of 300 counts/channel has been added. We attribute the background to electrons that scatter around the collimations slits. The $1 \mu \mathrm{m}$ slit also gave a single slit diffraction pattern seen in Fig. 6. The theoretical curve that accompanies this diffraction pattern uses the same parameters as the $100 \mathrm{~nm}$ theory, but an adjusted background. The $2 \mu \mathrm{m}$ slit gave no discernable diffraction pattern.

By seeing single slit diffraction patterns we have shown that it is possible to get diffraction limited collimation at relatively low electron energies, $\sim 500 \mathrm{eV}$. This collimation is not possible using laser machined molybdenum air slits due to the fact that they are $5 \mu \mathrm{m}$ wide, not perfectly uniform, and seem to have a tendency to have a small lensing effect on the beam. The FIB machined slits do degrade after continued electron exposure, as seen in the quality and collimation of the electron beam. The degradation is negligible for the first $\sim 100 \mathrm{~h}$ of use at illuminations that are typical for counting experiments. The degraded slits still produce better collimation than using a $5 \mu \mathrm{m}$ air slit.

\section{B. Electron diffraction from $100 \mathrm{~nm} \mathrm{Si/Ni} \mathrm{gratings}$ coated with Au/Pd}

Diffraction patterns at 100 and $900 \mathrm{eV}$ were taken using a $100 \mathrm{~nm}$ periodicity grating with an open fraction of $\sim 50 \%$ (see Fig. 7). The major difference between the two different energies is the behavior of the beam and diffraction peak widths. At $900 \mathrm{eV}$ there is a little difference between the raw beam width and the different diffraction order widths. At $100 \mathrm{eV}$, however, there is a noticeable difference between the beam widths and the diffraction order widths. This broadening of the diffraction peaks at lower energies is affected by the type of metallic coatings on the grating and modeled theoretically by the random potential. A more detailed discussion is presented in Sec. IV D. One thing that should be noted is that the collimation of our electron beam (especially

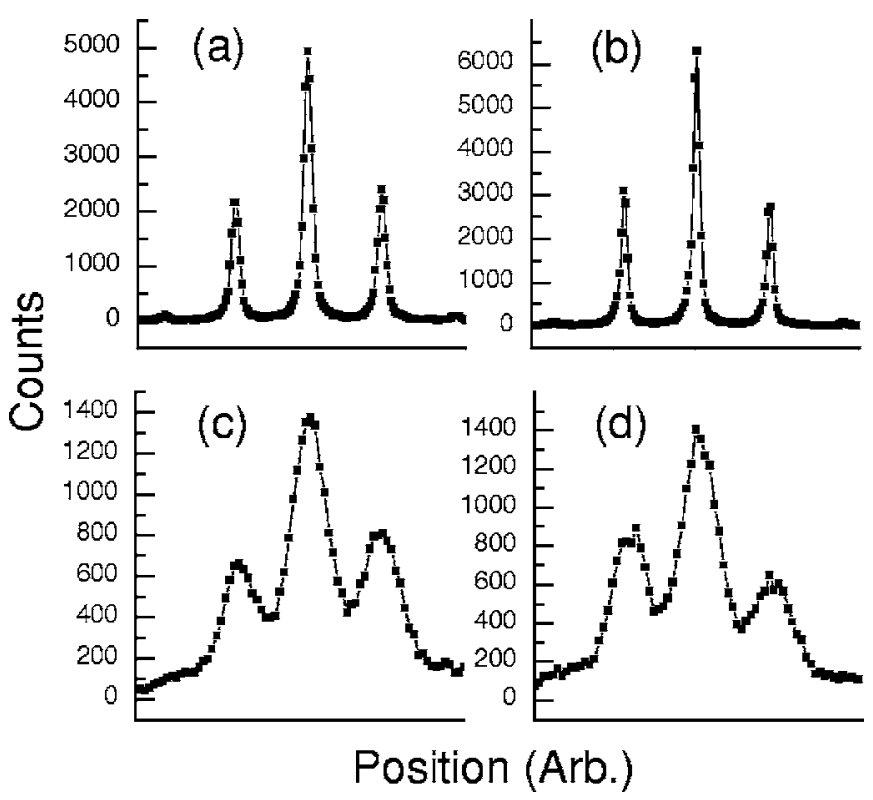

FIG. 8. Diffraction patterns taken at $\theta= \pm 5^{\circ}$ of grating tilt. Graph (a) is $\theta$ $=-5^{\circ}$ for $900 \mathrm{eV}$, (b) is $\theta=+5^{\circ}$ for $900 \mathrm{eV}$, (c) is $\theta=-5^{\circ}$ for $100 \mathrm{eV}$, and (d) is $\theta=+5^{\circ}$ for $100 \mathrm{eV}$. These diffraction patterns taken at $\theta= \pm 5^{\circ}$ have approximately the largest asymmetries of all the tilt angles.

at the lower energies) is somewhat less well collimated as compared to our previous work. ${ }^{27}$ This reduction in collimation is due to the collimation slit, which has deteriorated after prolonged electron beam exposure.

Energies of 100 and $900 \mathrm{eV}$ were chosen because these are near the limits of our current electron gun. The theoretical curve plotted is the result of our path integral calculation. The parameters used in the path integral calculations are discussed in Sec. IV C. The agreement between experiment and theory is reasonably good. This indicates that there is no evidence in any of our diffraction patterns of secondary electrons or inelastic scattering from the grating. The reason why small asymmetries are seen between the $+/-$ first orders at $\theta=0^{\circ}$ is not fully understood. They may be due to asymmetries along the beam path, caused by the electron beam traveling closer or further from different metal surfaces in the apparatus. The asymmetry is minimized by adjusting the path of the beam by moving the collimation slits.

\section{Image charge potential measurement}

While the diffraction patterns themselves can provide some insight into electron-grating interactions, rocking curves provide more detailed data that quantify electrongrating interactions. A rocking curve is made by observing the electron counts in a particular diffraction order versus grating tilt angle. The grating is rotated about an axis that is parallel to the grating bars and perpendicular to the electron beam (see Fig. 4). The reason a rocking curve is sensitive to the electron-grating interactions is that as the grating is rotated the distance between the electrons and the grating bar wall varies.

Rocking curves are created at electron energies of 100 and $900 \mathrm{eV}$. Several different diffraction patterns shown in Fig. 8 are typical to those that the rocking curves are con- 


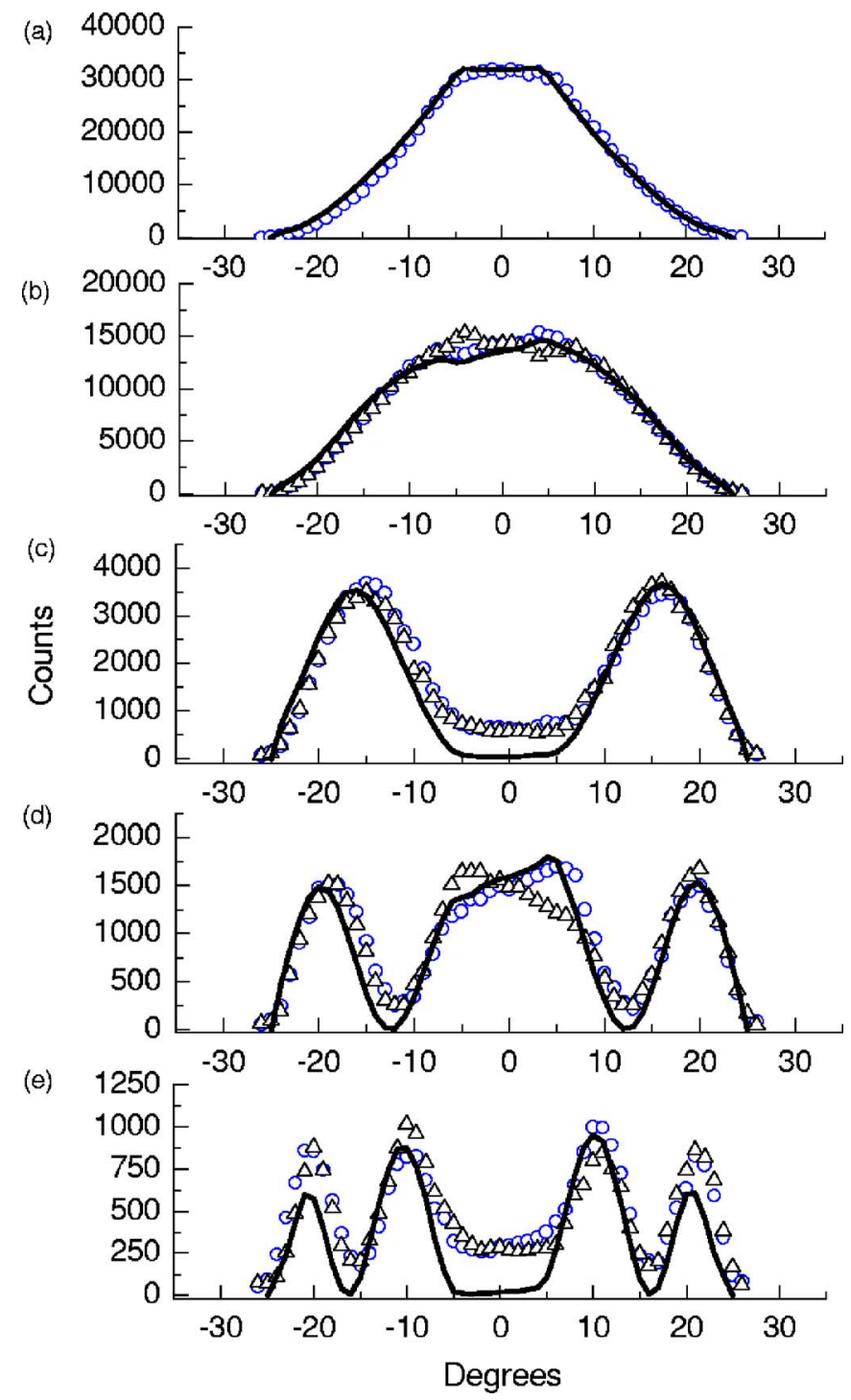

FIG. 9. (Color online) Rocking curves for $900 \mathrm{eV}$. The electron transmission into a particular order is given as a function of grating tilt angle. Graph (a) is zeroth order, (b) is the first order, (c) is the second order, (d) is the third order, and (e) is the fourth order. The squares and circles are the $+/-$ orders. The solid line is the model with image charge potential, random potential, and geometry of the grating included.

structed from. To construct the rocking curves, diffraction patterns are taken at $1^{\circ}$ increments from a $-26^{\circ}$ tilt angle to $26^{\circ}$ (Fig. 9). We determine the electron counts in a particular diffraction order by integrating over $60 \mu \mathrm{m}$. This is about five times the peak width.

For $100 \mathrm{eV}$ the data were taken with different parameters. Since both the diffraction angle and the diffraction peak widths for $100 \mathrm{eV}$ are larger, a wider $(120 \mu \mathrm{m})$ third slit was used and the diffraction patterns are still resolved. At the same time the wider slit compensates the lower count rate at $100 \mathrm{eV}$. Again 53 diffraction patterns were taken, and the count rate in a particular diffraction order was found by integrating over $50 \mu \mathrm{m}$ (see Fig. 10).

The calculation of the theoretical curves that accompany the experimental rocking curves exactly mimics the experimental circumstances. It is also important to note that both the 100 and the $900 \mathrm{eV}$ use the same grating parameters,

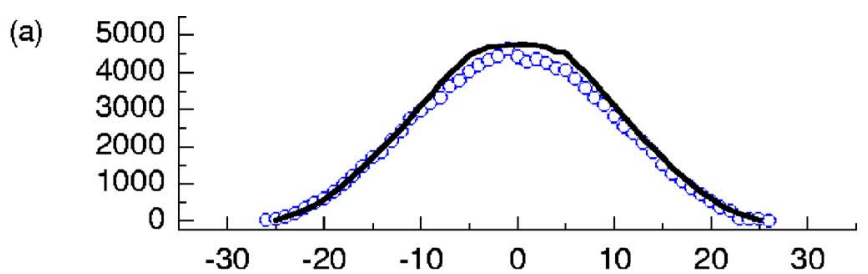

(b)
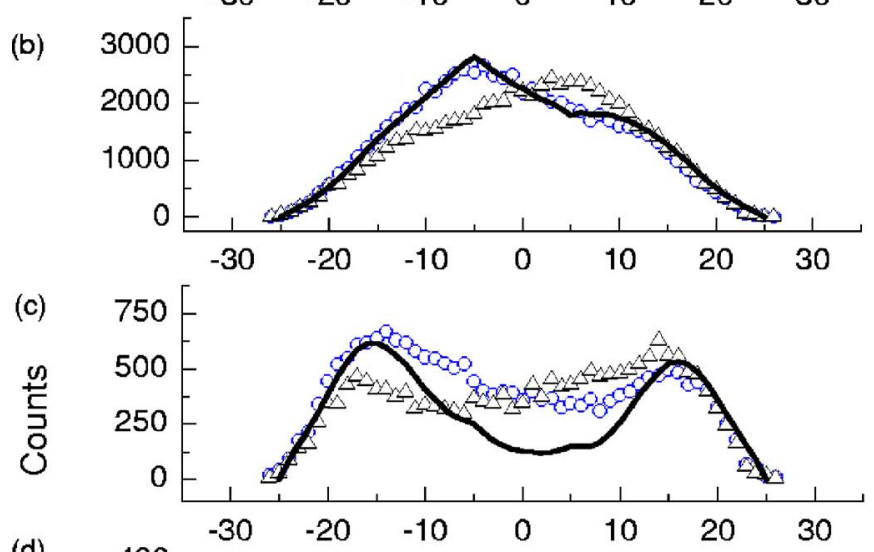

(d)

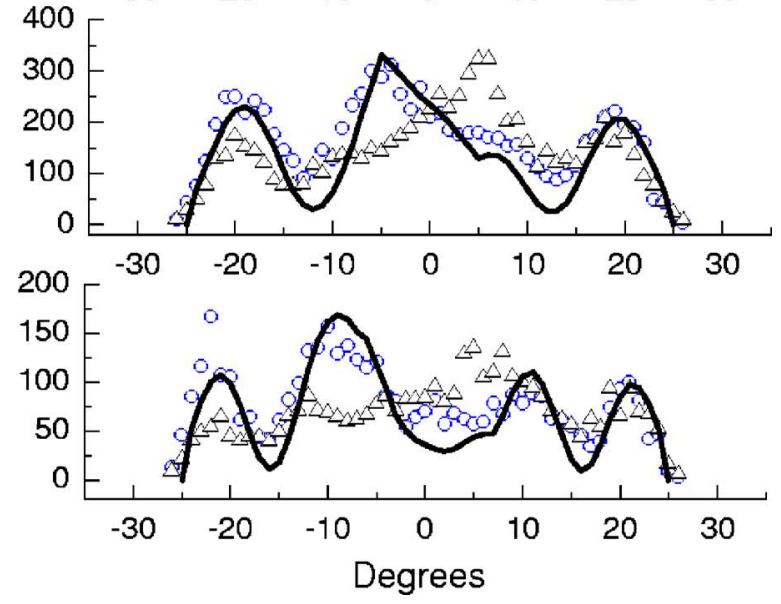

FIG. 10. (Color online) Rocking curves for $100 \mathrm{eV}$. The electron transmission into a particular order is given as a function of grating tilt angle. Graph (a) is zeroth order, (b) is the first order, (c) is the second order, (d) is the third order, and (e) is the fourth order. The squares and circles are the $+/-$ orders. The solid line is the model with image charge potential, random potential, and geometry of the grating included.

including the random potential and image charge strength. The calculations include the interaction time in these potentials, which scales linearly with electron velocity.

In the theory a first slit of $w_{1}=7.5 \mu \mathrm{m}$, second slit of $w_{\mathrm{ss}}=2 \mu \mathrm{m}$, and a detection slit (third) of $w_{3}=5 \mu \mathrm{m}$ were used. The gratings parameters used were periodicity $d$ of $100 \mathrm{~nm}$, slit width $w$ of $47.5 \mathrm{~nm}$, grating thickness $l$ of $122 \mathrm{~nm}$, and a bevel angle $\phi$ of $4.80^{\circ}$. The grating periodicity $d$ is well known due to the grating construction process, while the parameters $w, l$, and $\phi$ are not known exactly. While these three parameters are not known exactly, they are limited to certain values and can be constrained due to the observed cutoff angle $\theta$ of the grating [Eq. (37)]. These values are within the specifications of the gratings.

The effective image charge strength used in all the fits shown in this paper was $q_{\text {eff }}=0.13_{-0.07}^{+0.13} e$. The errors are estimated by choosing appropriate grating parameters and then comparing how different image charge strengths affect the corresponding fits. We should note here that our value for 


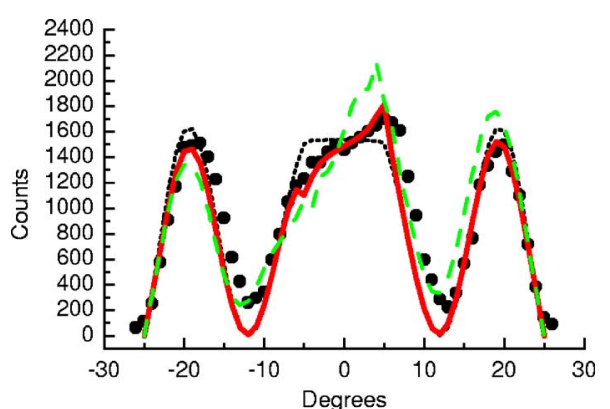

FIG. 11. (Color online) Rocking curves for $900 \mathrm{eV}$ third order. The electron transmission into the third order is given as a function of grating tilt angle. The solid circles are data. The solid line is the model with $q_{\text {eff }}=0.13 e$, the dotted line is with $q_{\text {eff }}=0$, and the dashed line is with $q_{\text {eff }}=e$.

image charge strength is reduced by a factor of 4 compared to that recently found by McMorran et al. ${ }^{20}$ This is reflected in the measured asymmetries in diffraction data, Fig. 8, which are smaller than those shown by McMorran et al. ${ }^{20}$ The largest asymmetries we get between the first orders (grating tilt $\theta= \pm 5^{\circ}$ ) are $\sim 15 \%$ of the first order height for $900 \mathrm{eV}$ and $\sim 25 \%$ for $100 \mathrm{eV}$, while McMorran et al. have asymmetries of more than $50 \%$ for their raw data at $500 \mathrm{eV} .^{20}$ These differences may partially be due to variations in grating parameters, and variations between individual coating runs. To highlight how different image charge strengths affect the diffraction patterns we plotted the third order rocking curve with different values of $q_{\text {eff }}$ (see Fig. 11). We should note that the asymmetries observed in both experiment and in the model are due to the combination of the image charge and the bevel angle of the grating bar wall. Both a nonzero image charge potential along with a nonzero bevel angle are needed to get an asymmetric rocking curve. 20,34

While a reduced effective image charge strength at higher electron energies was not observed, such a reduction could have been motivated by the fact that the plasmon frequency and the inverse of the interaction time are of the same order. At higher electron energies the image charge might not have sufficient time to form, therefore reducing its strength. The absence of a reduced image charge strength could be explained due to that fact that we may not have reached a high enough electron energy for this effect to be observable. Another possible reason is that our experimental error bars were too large to make this effect observable.

\section{Random potentials: Electron diffraction from gratings with different coatings}

To investigate the broadening of the diffraction peaks the effect of different metallic coatings was investigated. For this study three different coating materials, along with two different coating thicknesses, were used. All beam and diffraction patterns were taken at an electron energy of $125 \mathrm{eV}$. This energy was chosen because the broadening is well pronounced, but at $125 \mathrm{eV}$ it is still fairly easy to get a diffraction pattern for all the different coatings. To quantify the amount of broadening that the grating introduces to the beams, the separate diffraction orders were fitted with a Gaussian function. From the fits the full width at half maxi-
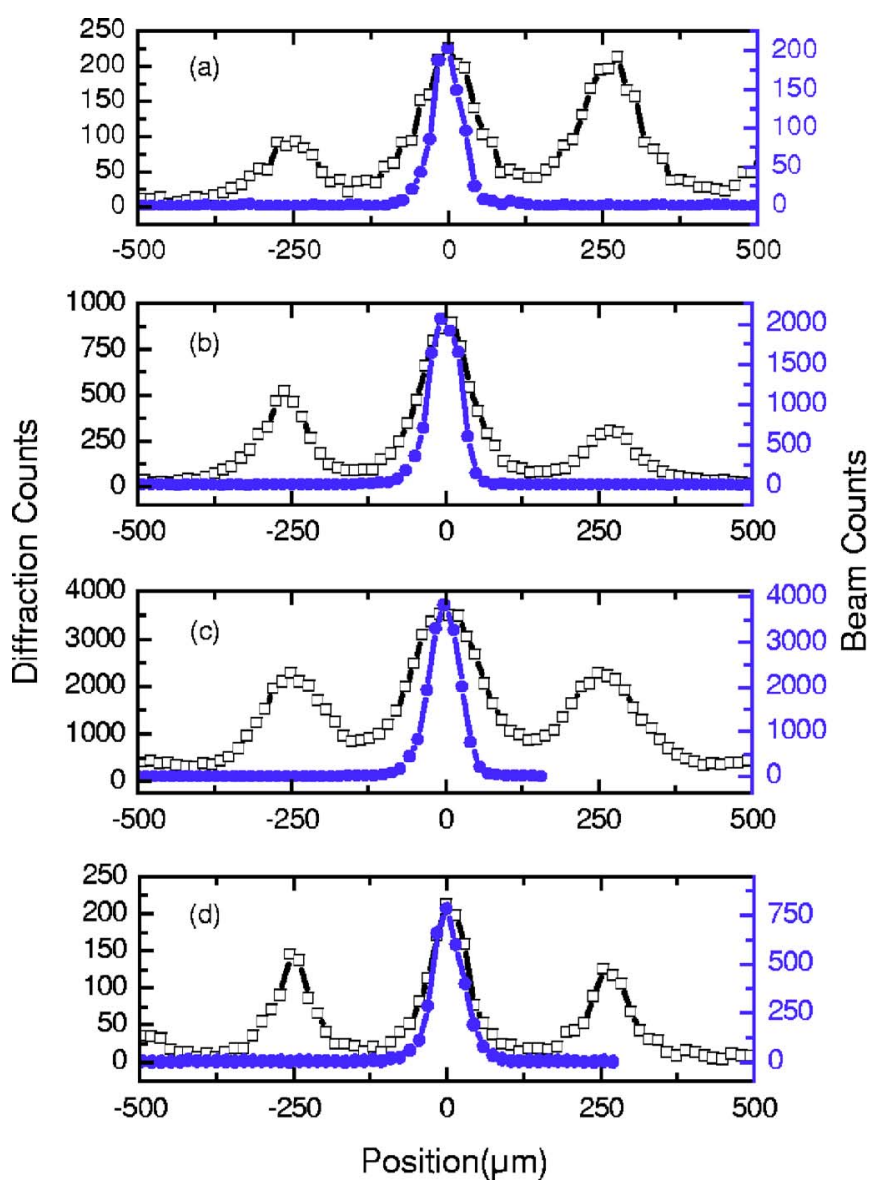

FIG. 12. (Color online) Diffraction patterns at $125 \mathrm{eV}$. The different coatings are (a) $16 \mathrm{~s}$ deposition of $\mathrm{Au} / \mathrm{Pd}$, (b) $48 \mathrm{~s}$ deposition of $\mathrm{Au} / \mathrm{Pd}$, (c) $\mathrm{Ti}$, and (d) Ni. The open squares are the diffraction pattern, and the solid dots are the associated beam data. The left vertical scales are for the diffraction pattern counts and the right vertical scales are for the beam counts.

mum $\left(\mathrm{FWHM}_{d}\right)$ was determined. To compare this to the electron beam width, $\left(\mathrm{FWHM}_{b}\right)$, a broadening factor $(\mathrm{BF})$ is defined as $\mathrm{BF}=\mathrm{FWHM}_{d} / \mathrm{FWHM}_{b}$.

The standard coating that we use is $60 \%$ gold and $40 \%$ palladium, with a thickness of approximately $2 \mathrm{~nm}$ on the front and back surfaces and $0.2 \mathrm{~nm}$ on the grating bar walls. This thickness of the metallic coating is not expected to give a uniform coating of the metal. The coating is put on the grating by sputtering for $32 \mathrm{~s}$. The first experiment to test the effect of coating on the peak broadening was to compare gratings with different $\mathrm{Au} / \mathrm{Pd}$ coating thicknesses. Sputtering times of 16 and $48 \mathrm{~s}$ were used (Fig. 12). The trend seems to be that a thicker coating of $\mathrm{Au} / \mathrm{Pd}$ causes slightly less broadening, which can be seen in Table I.

Different metal coatings (nickel, titanium, and aluminum) were applied to separate silicon nitride gratings. Nickel produced a clear improvement over the $\mathrm{Au} / \mathrm{Pd}$ coated gratings (see Fig. 12). The titanium coated grating had very similar broadening effects as the $\mathrm{Au} / \mathrm{Pd}$ coated gratings and the Al coated grating gave no diffraction pattern.

The $\mathrm{Al}$ and Ti coatings may have oxidized when stored in air, and this may have been a factor in their producing increased peak widths. Ni seems to be the best coating on the nanofabricated gratings for electron diffraction. One aspect 
TABLE I. Broadening factors (BFs) as a function of different metallic coatings, at $125 \mathrm{eV}$ electron energy. The broadening factor is defined as $\mathrm{BF}$ $=\mathrm{FWHM}_{d} / \mathrm{FWHM}_{b}$, the ratio of the full width at half maximum of the diffraction peaks $\mathrm{FWHM}_{d}$, over the full width at half maximum of the beam $\mathrm{FWHM}_{b}(\mu \mathrm{m})$. The symbol $\eta$ denotes the open fraction of the grating which is defined as the slit width $w$ divided by the periodicity $d$, where $d$ is $100 \mathrm{~nm}$.

\begin{tabular}{lcccc}
\hline \hline \multicolumn{1}{c}{ Coating } & $\mathrm{BF}$ & $\mathrm{FWHM}_{b}$ & $\mathrm{FWHM}_{d}$ & $\eta$ \\
\hline $16 s \mathrm{Au} / \mathrm{Pd}$ & 2.59 & 53.8 & 139.1 & 53 \\
$48 \mathrm{~s} \mathrm{Au} / \mathrm{Pd}$ & 2.14 & 57.7 & 123.7 & 56 \\
$\mathrm{Ti}$ & 2.71 & 57.7 & 156.6 & 53 \\
$\mathrm{Ni}$ & 1.63 & 57.7 & 93.8 & 48 \\
\hline \hline
\end{tabular}

of the Ni coated grating was that there was no evidence of the magnetic properties having any effect on the diffraction patterns.

This broadening of the individual diffraction peaks seems to be related to both the thickness of the coating and the type of metallic coating used. To support our use of a random potential model to explain the broadening we had one of the Au coated substrates examined by a scanning tunneling microscope (STM). The picture taken by the STM was not on the grating bars, but the substrate frame.

The STM picture shows protrusions on the substrate. The typical protrusion separation has a length scale on the order of a few hundred nanometers (see Fig. 13). Our random potential model has this same order of magnitude for the characteristic sizes of the potentials. The sharp demarcation between the protrusions and the surrounding material could lead to contact potentials. In our random potential model we used interaction strengths of $0.35 \mathrm{eV}$ consistent with contact potentials. As discussed in Sec. II D, the broadening of the diffraction orders cannot be explained by the $\Delta E$ in the electron beam, nor can the broadening be explained by a uniformity problem with the grating, as will be discussed in the next section.

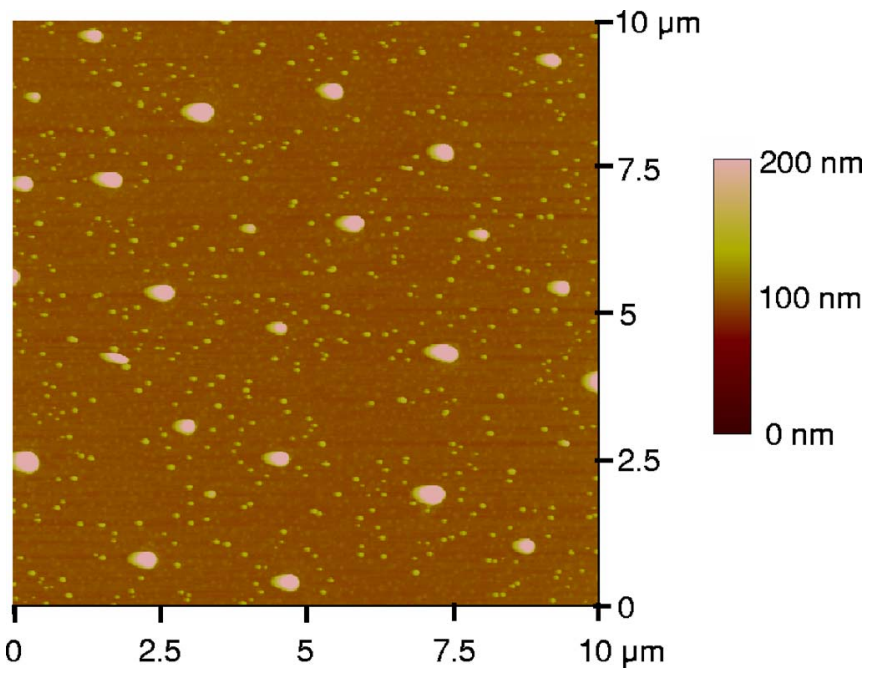

FIG. 13. (Color online) STM picture of the coated $\mathrm{Si}_{3} \mathrm{~N}_{4}$ substrate. The lighter regions are protrusions on the substrate.

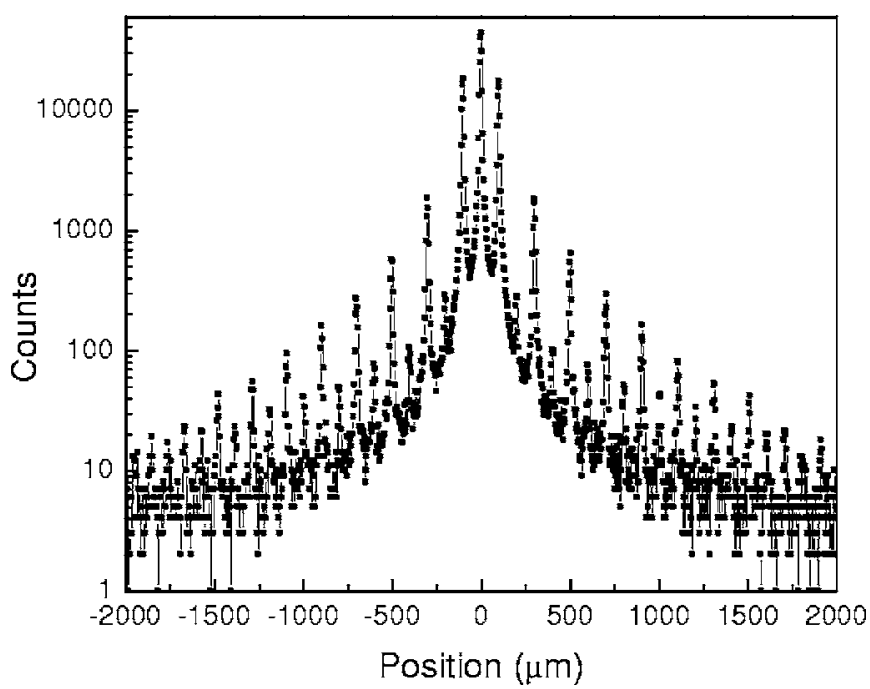

FIG. 14. Electron diffraction at $900 \mathrm{eV}$. Both plus and minus orders are shown out to approximately 20 th order.

\section{E. Grating quality}

When Fig. 7 is expanded vertically (logarithmic scale) and horizontally, a diffraction pattern out to the 20th order can clearly be observed (Fig. 14). This illustrates the high quality of the nanofabricated gratings.

From such detailed diffraction patterns the structure of the gratings can be studied. Grisenti et al. used similar gratings (but uncoated) to diffract $\mathrm{He}$ and determined average slit widths, bar profiles, surface roughness of the grating, and statistical randomness in the individual slit positions. ${ }^{48}$ They were able to obtain very good agreement between theory and experiment out to the sixth order. Those gratings were used to construct an interferometer for atoms and molecules. The question addressed in this paper is if electron interferometers can be constructed with these gratings. This is nontrivial due to the fact that an electron is light and charged. The charge can induce strong interactions and the low mass of the electron can intensify the effect of the interaction. The randomness in the grating parameters measured by Grisenti et al. did not impede the construction of atom/molecule interferometers and is not expected to do so for electrons. However, the added difficulty of electron-grating interaction due to the charge of the electron must be addressed.

In particular, the effect of electron-grating interaction is important for the coherence properties of the electron wave. From the diffraction pattern in Fig. 14 we can make straightforward qualitative observations concerning the transverse and longitudinal coherence lengths. As discussed in previous sections the peak width of each diffraction order is related to the transverse coherence length. At $900 \mathrm{eV}$ there is a little broadening, so the grating does not affect the electron wave in this respect. This can be quantified by examining the diffraction pattern shown in Fig. 14. The peak widths of higher orders can be affected by the longitudinal coherence length (according to the usual dispersive nature of gratings), and we estimate that for the 20th order, $\Delta \theta_{\text {diff }} / \theta_{\text {diff }} \simeq 0.1$. Assuming that all broadening is due to the energy spread of the electron source we can then use Eq. (37) to find that $\Delta E \simeq 5 \mathrm{eV}$, at an electron energy of $900 \mathrm{eV}$. This corresponds to a lower limit 


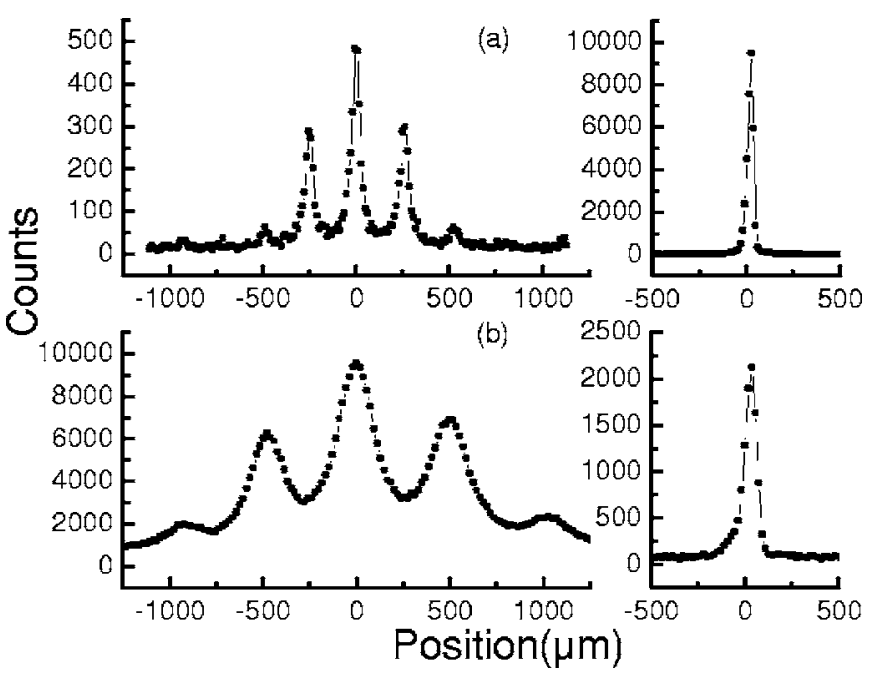

FIG. 15. Electron beam diffraction. Electron diffraction from Ni coated gratings presented as a function of position for energies of (a) 125 and (b) $50 \mathrm{eV}$. The beam profile without a diffraction grating is shown for comparison.

on the longitudinal coherence length of $\sim 10 \mathrm{~nm}$. This is comparable to the longitudinal coherence length $(17 \mathrm{~nm})$ of a working low energy biprism electron interferometer. ${ }^{49}$

\section{F. Lowest energy diffraction patterns}

The highest quality low energy electron diffraction patterns that we have observed with these nanofabricated gratings are shown in Fig. 15. These patterns were taken with a $\mathrm{Ni}$ coated grating. With other coatings we could not reach $50 \mathrm{eV}$ electron energies and still see diffraction. The $50 \mathrm{eV}$ diffraction has first order diffraction peaks that are located almost $0.5 \mathrm{~mm}$ from the zeroth order. This large separation could be used to construct a wide angle electron interferometer. In our current dedicated electron beam system $50 \mathrm{eV}$ is the lowest energy at which we can obtain a collimated beam. A better designed electron gun, improved shielding, a different geometry of the slit placement, and deflection plates would be needed to reach lower energies.

\section{SUMMARY AND DISCUSSION}

We have shown that it is possible to observe electron diffraction from freestanding nanofabricated gratings in the energy range of 50-900 eV. The quality of these diffraction patterns is a function of the incoming beam collimation (which is directly related to the transverse coherence length), and the amount of dephasing due to random potentials on the grating. Our ability to construct diffraction limited electron beams, by using FIB machined slits in $100 \mathrm{~nm}$ substrates, increases our transverse coherence length to the optimal amount for resolving the diffraction patterns. We have also presented a simple random potential model that seems to replicate the decrease transverse coherence length we see in our experimental data. The image charge potential was examined in more detail by using the rocking curve method. Rocking curves are one way to observe asymmetries in the different orders, which was due to the presence of the image charge potential and the bevel angle of the individual grating bars. These asymmetries were also found when this system was modeled, which allowed us to determine the strength of the image charge potential. The random potential model motivated us to investigate the effect of different metallic coatings on the broadening of the diffraction peaks. This investigation showed that $\mathrm{Ni}$ coated gratings produced the best resolved patterns, which lead to our lowest energy diffraction patterns at $50 \mathrm{eV}$.

The measured image potential strength is $\sim 15 \%$ of that expected for a charge next to an ideal metallic wall. This indicates that there is some reduction in the image charge strength which would be interesting to investigate in more detail. The rocking curve method for determining the strength of the image charge works for electrons and is good enough to attempt time-dependent studies. In this context we observe that the interaction time $\left(10^{-14} \mathrm{~s}\right)$ is of the order of the inverse plasma frequencies of typical metals. A timedependent permittivity study may be relevant to measuring the response functions of plasmons as a characterization of metals. ${ }^{50}$ This method of determining the permittivity is not limited to metal coated $\mathrm{Si}_{3} \mathrm{~N}_{4}$ gratings and could be done with other materials. At present, however, the only other material that these gratings can be made from is Au. As was shown in our recent publication the random potential model would not prohibit the functioning of a three grating electron interferometer. ${ }^{27}$ Our investigation of different coatings shows that this random potential can be reduced.

\section{ACKNOWLEDGMENTS}

The authors thank Tim Savas for production of the gratings. This material is based upon work supported by the National Science Foundation under Grant No. 0112578.

${ }^{1}$ G. Möllenstedt and H. Düker, Naturwiss. 42, 41 (1955).

${ }^{2}$ H. Kiesel, A. Renz, and F. Hasselbach, Nature (London) 418, 392 (2002).

${ }^{3}$ R. G. Chambers, Phys. Rev. Lett. 5, 3 (1960).

${ }^{4}$ A. Tonomura et al., Phys. Rev. Lett. 48, 1443 (1982).

${ }^{5}$ A. Tonomura, N. Osakabe, T. Matsuda, T. Kawasaki, J. Endo, S. Yano, and H. Yamada, Phys. Rev. Lett. 56, 792 (1986).

${ }^{6}$ F. Hasselbach and M. Nicklaus, Phys. Rev. A 48, 143 (1993).

${ }^{7}$ F. Hasselbach, H. Kiesel, and P. Sonnentag, Ann. Fond. Louis Broglie 29, 857 (2004).

${ }^{8}$ R. C. Forrey, A. Dalgarno, and J. Schmiedmayer, Phys. Rev. A 59, R942 (1999).

${ }^{9}$ H. Ulys, J. D. Perreault, and A. D. Cronin, Phys. Rev. Lett. 95, 150403 (2005).

${ }^{10} \mathrm{~K}$. Hornberger, S. Uttenthaler, B. Brezger, L. Hackermüller, M. Arndt, and A. Zeilinger, Phys. Rev. Lett. 90, 160401 (2003).

${ }^{11}$ B. E. Allman, A. Cimmino, A. G. Klein, and G. I. Opat, Phys. Rev. Lett. 68, 2409 (1992).

${ }^{12}$ G. Badurek, H. Weinfurter, R. Gahler, A. Kollmar, S. Wehinger, and A. Zeilinger, Phys. Rev. Lett. 71, 307 (1993).

${ }^{13}$ M. Nicklaus, thesis, Eberhards-Karls-Universität zu Tübingen, 1989.

${ }^{14}$ D. K. Ross, Nuovo Cimento Soc. Ital. Fis., A 110A, 571 (1997).

${ }^{15}$ C. Davisson and L. H. Germer, Phys. Rev. 30, 705 (1927).

${ }^{16}$ L. Marton, J. A. Simpson, and J. A. Suddeth, Phys. Rev. 85, 490 (1953).

${ }^{17}$ L. Marton, J. A. Simpson, and J. A. Suddeth, Rev. Sci. Instrum. 25, 1099 (1954).

${ }^{18}$ V. P. Holl, Optik (Stuttgart) 30, 116 (1969).

${ }^{19}$ Y. Ito, A. L. Bleloch, and L. M. Brown, Nature (London) 394, 49 (1998).

${ }^{20}$ B. McMorran, J. D. Perreault, T. Savas, and A. Cronin, Ultramicroscopy 106, 356 (2006).

${ }^{21}$ D. L. Freimund, K. Aflatooni, and H. Batelaan, Nature (London) 413, 142 (2001).

${ }^{22}$ C. Jönsson, Z. Phys. 161, 454 (1961). 
${ }^{23}$ T. A. Savas, S. N. Shah, M. L. Schattenburg, J. M. Carter, and H. I. Smith, J. Vac. Sci. Technol. B 13, 2732 (1995).

${ }^{24}$ M. S. Chapman, C. R. Ekstrom, T. E. Hammond, R. A. Rubenstein, J. Schmiedmayer, S. Wehinger, and D. E. Pritchard, Phys. Rev. Lett. 74, 4783 (1995).

${ }^{25}$ R. E. Grisenti, W. Schöllkopf, J. P. Toennies, G. C. Hegerfeldt, T. Köhler, and M. Stoll, Phys. Rev. Lett. 85, 2284 (2000).

${ }^{26}$ M. Arndt, O. Nairz, J. Vos-Andreae, C. Keller, G. v. d. Zouw, and A Zeilinger, Nature (London) 401, 680 (1999).

${ }^{27}$ G. Gronniger, B. Barwick, H. Batelaan, T. Savas, D. Pritchard, and A Cronin, Appl. Phys. Lett. 87, 124104 (2005).

${ }^{28}$ A. Rivacoba, N. Zabala, and J. Aizpurua, Prog. Surf. Sci. 65, 1 (2000).

${ }^{29}$ H. Winter, J. Phys.: Condens. Matter 8, 10149 (1996).

${ }^{30}$ J. P. Muscat and D. M. Newns, Surf. Sci. 64, 641 (1976).

${ }^{31}$ P. M. Echenique and J. B. Pendry, J. Phys. C 8, 2936 (1975).

${ }^{32}$ C. S. Adams, M. Sigel, and J. Mlynek, Phys. Rep. 240, 143 (1994).

${ }^{33}$ E. Hecht, Optics, 4th ed. (Addison-Wesley, San Francisco, CA, 2002).

${ }^{34}$ A. D. Cronin and J. D. Perreault, Phys. Rev. A 70, 043607 (2004).

${ }^{35}$ R. P. Feynman, Rev. Mod. Phys. 20, 367 (1948).

${ }^{36} \mathrm{Q}$. A. Turchette, Bsc, thesis, Massachusetts Institute of Technology, 1991

${ }^{37}$ J. W. Goodman, Introduction to Fourier Optics (McGraw-Hill, San Fran- cisco, CA, 1968).

${ }^{38}$ R. E. Grisenti, W. Schöllkopf, J. P. Toennies, G. C. Hegerfeldt, and T. Köhler, Phys. Rev. Lett. 83, 1755 (1999).

${ }^{39}$ B. Brezger, L. Hackermüller, S. Uttenthaler, J. Petschinka, M. Arndt, and A. Zeilinger, Phys. Rev. Lett. 88, 100404 (2002).

${ }^{40}$ J. D. Perreault and A. D. Cronin, Phys. Rev. A 71, 053612 (2005).

${ }^{41}$ M. K. Park, H. N. Kim, K. S. Lee, S. S. Baek, E. S. Kang, Y. K. Baek, and D. K. Kim, Key Eng. Mater. 287, 247 (2005).

${ }^{42}$ J. D. Jackson, Classical Electrodynamics, 3rd ed. (Wiley, New York, 1999).

${ }^{43}$ R. Shakeshaft and L. Spruch, Phys. Rev. A 22, 811 (1980).

${ }^{44}$ J. R. Anglin and W. H. Zurek, e-print quant-ph/9611049.

${ }^{45}$ B. A. Rose, Phys. Rev. 44, 585 (1933).

${ }^{46}$ R. Brühl, A. Kalinin, O. Kornilov, J. P. Toennies, G. C. Hegerfeldt, and M. Stoll, Phys. Rev. Lett. 95, 063002 (2005).

${ }^{47}$ O. Nairz, M. Arndt, and A. Zeilinger, Phys. Rev. A 65, 032109 (2002).

${ }^{48}$ R. E. Grisenti, W. Schöllkopf, and J. P. Toennies, Phys. Rev. A 61, 033608 (2000).

${ }^{49}$ M. Nicklaus and F. Hasselbach, Phys. Rev. A 48, 152 (1993).

${ }^{50}$ P. A. Dowben, Surf. Sci. Rep. 40, 151 (2000). 